\title{
Stability of Time-Delay Systems with Non-Small Delay
}

\author{
Frédéric Gouaisbaut and Dimitri Peaucelle \\ LAAS-CNRS - Univ. de Toulouse \\ 7 avenue du Colonel Roche, 31077 Toulouse, FRANCE \\ \{gouaisbaut, peaucelle\}@laas.fr
}

March 8, 2006

\begin{abstract}
This paper is dedicated to the stability of linear time-delay system for a non small delay $h$. This is motivated by the fact that in some cases introducing a delay in the loop may stabilize a system. Compared to previously derived results the methodology is totally new and the resulting LMI formulas are original contributions. The derived criteria are based on Taylor series of the delay operator for which the Lagrange remainder is treated as a bounded uncertainty. A sequence of results with decreasing conservatism are obtained applying quadratic separation. An extension to robust analysis with respect to uncertain delay is also derived. A numerical example illustrates the efficiency.

Keywords: Linear time-delay systems, Stability, LMIs.
\end{abstract}

\section{Introduction}

During the last decade, the stability analysis of time-delay systems with LMI based methods have attracted a large number of researchers $[9,21,5,10,20,4]$. The criteria developed can be classified into two major categories: the delay independent case and the delay dependent case. The first category assumes the delay unknown and possibly unbounded. The criteria therefore does not depend on the size of the delay. In the second category, most delay-dependent results (see [8] and references therein) apply to systems stable without delays and look for the maximal delay that preserves stability. Nevertheless, as it has been shown in $[1,3,15]$ and references therein, there exist systems that are unstable for zero delay and becomes stable only for some strictly positive values of the delay.

This paper addresses this problem of proving the stability of a linear time-delay system for a given delay $h$ without assuming the system to be stable for $h=0$. At this time several papers deal with this problem of non-small delays (also known as interval delays). The approaches developed in these are quite distinct. In [13], necessary and sufficient conditions are found using matrix pencil framework. These results are analytic and compared with LMI-based results have therefore few possible derivations for robustness purpose, performance analysis or design. LMI sufficient condition are as well derived using a modification of the complete Lyapunov-Krasovskii functional developed by [12]. Another result is that of [4] in which the author attempt to give LMI-based results assuming variations $\tau$ around a nominal delay $h_{n o m}$ for which the system is supposed stable. A new Lyapunov-Krasovskii functional is introduced to take into account that $h_{n o m}$ may be different from zero. Unfortunately, one may show that the Lyapunov-Krasovskii functional proves simultaneously stability of the system for delays included in $\left[0 h_{n o m}\right]$. Their results is therefore identical to those compared in [8]. A third approach described in [9], relies on the discretization of a complete Lyapunov-Krasovskii functional. Results are LMI-based and are asymptotically exact as the discretization is refined. A fourth and significant result of [14] is based on Padé approximation. Results rely on two steps: one is analytical and tunes the Padé approximation for the considered delay interval; the second is a sufficient LMI formulation for an artificially created uncertain system without delays. As the order of the Padé approximation grows the method is proved to have arbitrarily small conservatism.

In the present paper an alternative way to prove the stability of time-delay systems using Taylor series and the quadratic separation formalism is exposed. It has the advantage to allow simple extensions for 
robustness issues and descriptor systems as demonstrated in [16]. One of such extensions is detailed in the present manuscript: robust stability with respect to uncertain delay bounded in an interval.

The concept of Topological Separation [17] is an alternative framework to Lyapunov Theory for proving stability of systems. In that framework, stability (or well-posedness) is defined with respect to some feedback connection and is proved at the expense of finding a separator function. Depending on the feedback connection modeling, this separator has direct relation with Lyapunov functionals. In case of linear systems, the separator may be chosen as a quadratic function as demonstrated in [11]. These last results where extended for descriptor systems in [16] and were applied to time-delay system stability analysis in the [8]. A closely related, alternative to the Lyapunov framework, approach can also be found in [21, 10]. In these, delay-dependent results on time-delay system stability are obtained applying small-gain methodology (which is a sub-case of the general quadratic separation) to an artificially modified "comparison" system that involves not only the Laplace operator $s$ and the delay operator $e^{-s h}$ but also some new combination of these two.

But these paper deal only with the usual delay-dependent case for which the system with zero delay has to be stable. To improve these results and handle the case of non-small delay, the contribution of the present paper is based on Taylor series representation of the delay operator. The Taylor series terms are taken up to a power $k$, all which elements are treated exactly, and the Lagrange remainder is treated as an uncertainty. As the maximal power $k$ grows the approximation due to over-bounding of the Lagrange remainder tends to zero hence reducing the conservatism. When taking trivial series up to the power $k=0$ well-known delay-independent results are recovered. Taking the Taylor series up to the power $k=1$ is proved equivalent to results of [8] for the delay-dependent case.

The paper is organized as follows. In the next section, some preliminaries are given and the result of quadratic separation is recalled. The third section is then devoted to the main delay-dependent LMI result for non-small delay. Section IV demonstrates how the former results extend to robust analysis with respect to uncertain delay bounded in an interval. The fifth section illustrates the theoretical results on classical academic examples. Finally the papers closes on some conclusions and prospectives.

\section{Preliminaries}

\subsection{Notations}

For a two symmetric matrices, $A$ and $B, A>(\geq) B$ means that $A-B$ is (semi-) positive definite. $A^{T}$ denotes the transpose of $A .1_{n}$ and $0_{m, n}$ denote the respectively the identity matrix of size $n$ and null matrix of size $m \times n$. If the context allows it the dimensions of these matrices are often omitted. For a given matrix $B \in \mathrm{R}^{m \times n}$ such that $\operatorname{rank}(B)=r$, we define $B^{\perp} \in \mathrm{R}^{n \times(n-r)}$ the right orthogonal complement of $B$ by $B B^{\perp}=0$ and $B^{\perp} B^{\perp T}>0$. The set of complex numbers of the right-half of the complex plane is denoted $\mathrm{C}^{+}=\left\{s \in \mathrm{C}: s+s^{*} \geq 0\right\}$. For block-diagonal matrices the following notation is adopted

$$
\operatorname{diag}\left(\begin{array}{c}
A \\
B \\
C
\end{array}\right)=\left[\begin{array}{ccc}
A & 0 & 0 \\
0 & B & 0 \\
0 & 0 & C
\end{array}\right] .
$$

\subsection{Review of quadratic separation}

Well-posedness of feedback systems provides a fertile framework for stability analysis of non-linear and uncertain systems. Major results for robust stability analysis has been given in [11] and references therein. The purpose of this section is to briefly recall some new tools on quadratic separation developed for robustness issues of descriptor systems [16], which is needed for the main theorem of this paper.

Consider two possibly non-square matrices $\mathcal{E}$ and $\mathcal{A}$ and an uncertain constant, complex valued, matrix $\nabla$ with appropriate dimensions that belongs to some set $\mathbb{W}$. For simplicity of notations we assume in the present paper that $\mathcal{E}$ is full column rank. We make no assumption on the uncertainty set $\mathbb{W}$. 


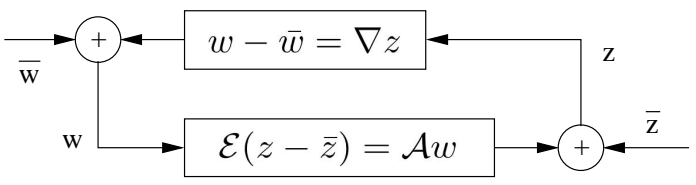

Figure 1: Feedback system

Theorem 1 [16] The uncertain feedback system of Figure 1 is well-posed if and only if there exists a Hermitian matrix $\Theta=\Theta^{*}$ satisfying both conditions

$$
\begin{gathered}
{\left[\begin{array}{cc}
\mathcal{E} & -\mathcal{A}
\end{array}\right]^{\perp *} \Theta\left[\begin{array}{ll}
\mathcal{E} & -\mathcal{A}
\end{array}\right]^{\perp}>0} \\
{\left[\begin{array}{cc}
1 & \nabla^{*}
\end{array}\right] \Theta\left[\begin{array}{c}
1 \\
\nabla
\end{array}\right] \leq 0, \quad \forall \nabla \in \mathbb{W} .}
\end{gathered}
$$

If $\mathcal{E}$ and $\mathcal{A}$ are real matrices, the equivalence still holds with $\Theta$ restricted to be a real matrix.

A simple corollary of that result is for LTI system stability analysis. The system $\dot{x}(t)=A x(t)$, where $x(t) \in \mathrm{R}^{n}$, is stable if $A$ has all its eigenvalues in the left half plane, which is equivalent to the wellposedness of the feedback system of Figure 1 with $\mathcal{E}=1_{n}, \mathcal{A}=A$ and $\mathbb{W}=\left\{s^{-1} 1_{n}, s^{-1} \in \mathrm{C}^{+}\right\}$. In that case, the separator may be chosen losslessly as

$$
\Theta=\left[\begin{array}{cc}
0 & -P \\
-P & 0
\end{array}\right], P \in \mathrm{R}^{n \times n}>0
$$

Another corollary for delay-independent stability analysis of time-delay systems reads as follows. Let the time-delay system:

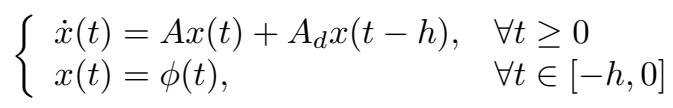

where $x(t) \in \mathrm{R}^{n}$ is the instantaneous state, $h$ is the constant delay, $\phi$ is the initial condition and $A, A_{d} \in$ $\mathrm{R}^{n \times n}$ are known constant matrices. Stability of that system whatever $h \geq 0$, can be recast as the wellposedness of the feedback system of Figure 1 with $\mathcal{E}=1_{2 n}$,

$$
\mathcal{A}=\left[\begin{array}{cc}
A & A_{d} \\
1_{n} & 0
\end{array}\right]
$$

and

$$
\mathbb{W}=\left\{\left[\begin{array}{cc}
s^{-1} 1_{n} & 0 \\
0 & e^{-s h} 1_{n}
\end{array}\right], s^{-1} \in \mathrm{C}^{+}, h \in \mathrm{R}^{+}\right\} .
$$

In that case, the separator may be chosen as

$$
\Theta=\left[\begin{array}{cccc}
0 & 0 & -P & 0 \\
0 & -Q & 0 & 0 \\
-P & 0 & 0 & 0 \\
0 & 0 & 0 & Q
\end{array}\right]
$$

with $P \in \mathrm{R}^{n \times n}>0$ and $Q \in \mathrm{R}^{n \times n}>0$. Such LMI results can be found in [10] (and references therein) and are known to be conservative due to the lossly choice of separators $\Theta$. They have been improved in [2] for approaching asymptotically non conservative delay-independent conditions.

In order to derive delay-dependent results another modeling of the time-delay system was proposed in [8]. Again the result is a corollary of Theorem 1 applied by taking

$$
\mathcal{E}=\left[\begin{array}{ccc}
1_{n} & 0 & 0 \\
0 & 1_{n} & 0 \\
1_{n} & 0 & -1_{n}
\end{array}\right], \mathcal{A}=\left[\begin{array}{ccc}
A & A_{d} & 0 \\
1_{n} & 0 & 0 \\
-1_{n} & 1_{n} & h 1_{n}
\end{array}\right]
$$


and

$$
\mathbb{W}=\left\{\left[\begin{array}{ccc}
s^{-1} 1_{n} & 0 & 0 \\
0 & e^{-s h} 1_{n} & 0 \\
0 & 0 & \frac{1-e^{-s h}}{s h} 1_{n}
\end{array}\right], s^{-1} \in \mathrm{C}^{+}\right\} .
$$

In that case, the separator may be chosen as

$$
\Theta=\left[\begin{array}{cccccc}
0 & 0 & 0 & -P & 0 & 0 \\
0 & -Q & 0 & 0 & 0 & 0 \\
0 & 0 & -R & 0 & 0 & 0 \\
-P & 0 & 0 & 0 & 0 & 0 \\
0 & 0 & 0 & 0 & Q & 0 \\
0 & 0 & 0 & 0 & 0 & R
\end{array}\right]
$$

with $P \in \mathrm{R}^{n \times n}>0, Q \in \mathrm{R}^{n \times n}>0$ and $R \in \mathrm{R}^{n \times n}>0$. Results derived in this way allow to handle the delay-dependent case, i.e. prove stability for all delays of $[0 h]$. Results for the case of non-small delay cannot be obtained at this point.

\subsection{Taylor series and Lagrange remainder}

The Taylor series representation of a function $f: \mathrm{R} \mapsto \mathrm{C}$ about the point $h_{0}$ is given by, [19],

$$
f(h)=\sum_{i=0}^{k-1} \frac{1}{i !} f^{(i)}\left(h_{0}\right)\left(h-h_{0}\right)^{i}+R_{k}(h)
$$

where $R_{k}$ is the Lagrange remainder that satisfies

$$
R_{k}(h)=\frac{1}{k !} f^{(k)}\left(h^{*}\right)\left(h-h_{0}\right)^{k}
$$

for some value $h_{k}^{*} \in\left[h_{0} h\right]$. Let any complex valued $s \in \mathrm{C}$ and consider the function $f(h)=e^{-s h}$, its Taylor series about the point $h_{0}=0$ writes as

$$
e^{-s h}=\sum_{i=0}^{k-1} \frac{1}{i !}(-s h)^{i}+R_{k}(h)
$$

and the Lagrange remainder is exactly

$$
R_{k}(h)=\frac{1}{k !}(-s h)^{k} e^{-s h_{k}^{*}}
$$

for some value $h_{k}^{*} \in[0 h]$. Note that the sequence of these remainders tends to zero as $r$ grows to infinity, and the Taylor series is absolutely convergent for all $h \in \mathrm{R}$ and $s \in \mathrm{C}$. These relations will be used in the following to derive results that take advantage of better knowledge of the delay operator. In particular, we can see that when $k=0$ the Taylor series involves only the operator $e^{-s h}$ as for delay-independent results, while the Taylor series taken up to the power $k=1$ involves the remainder $R_{1}$ that also writes as

$$
R_{1}(h)=\int_{0}^{h} \frac{1}{0 !}(h-\tau)^{0} f^{(1)}(\tau) d \tau=1-e^{-s h}
$$

and recalls the operator used for the delay-dependent case. The contribution of the paper relies on the use of the Taylor series taken up to further powers. 


\section{Main result}

In order to derive the main result, the Taylor series described above are translated in terms of relations between signals. As a start, define the operator

$$
\delta_{h}: v(t) \rightarrow \delta_{h}[v(t)]=v(t-h)
$$

which transfer function is $e^{-s h} 1$ and is norm-bounded $\left|e^{-s h}\right| \leq 1$ for all $s \in \mathrm{C}^{+}$and $h \geq 0$. Then consider the Taylor series up to the power $k=1$ to get

$$
x(t-h)=x(t)-h r_{1}(t)
$$

with $r_{1}(t)=\delta_{h_{1}^{*}}[\dot{x}(t)]$ for some $h_{1}^{*} \in[0 h]$. The property of the taylor series of function $f(h)=e^{-s h}$ implies that one has as well

$$
r_{1}(t)=\dot{x}(t)-\frac{h}{2} r_{2}(t)
$$

with $r_{2}(t)=\delta_{h_{2}^{*}}[\ddot{x}(t)]$ for some $h_{2}^{*} \in[0 h]$ and recursively one gets the relations for all $i=1 \ldots k$

$$
r_{i-1}(t)=x^{(i-1)}(t)-\frac{h}{i} r_{i}(t)
$$

with $r_{i}(t)=\delta_{h_{i}^{*}}\left[x^{(i)}(t)\right]$ for some $h_{i}^{*} \in[0 h]$. Since the delay $h$ is assumed constant, all these equations remain valid when taking their time derivatives. That is for all $i=1 \ldots k$ and for all $j=0 \ldots k-i$

$$
r_{i-1}^{(j)}(t)=x^{(i-1+j)}(t)-\frac{h}{i} r_{i}^{(j)}(t)
$$

with $r_{i}^{(j)}(t)=\delta_{h_{i}^{*}}\left[x^{(i+j)}(t)\right]$. All these intricate relations may be recast more simply. For that, define an extended state vector that includes time derivatives up to the degree $k-1$ :

$$
\tilde{x}(t)=\left(\begin{array}{c}
x^{(k-1)}(t) \\
\vdots \\
\dot{x}(t) \\
x(t)
\end{array}\right)
$$

and the following vectors $(i=1 \ldots k)$

$$
\tilde{z}_{i}(t)=\left(\begin{array}{c}
x^{(k)}(t) \\
\vdots \\
x^{(i+1)}(t) \\
x^{(i)}(t)
\end{array}\right) \quad, \quad \tilde{r}_{i}(t)=\left(\begin{array}{c}
r_{i}^{(k-i)}(t) \\
\vdots \\
\dot{r}_{i}(t) \\
r_{i}(t)
\end{array}\right) .
$$

Define as well the following matrices $(i=1 \ldots k-1)$

$$
\begin{aligned}
& E_{i}=\left[\begin{array}{ll}
0_{n(k-i), n} & 1_{n(k-i)}
\end{array}\right] \in \mathrm{R}^{n(k-i) \times n(k-i+1)} \\
& F_{i}=\left[\begin{array}{ll}
1_{n(k-i)} & 0_{n(k-i), n i}
\end{array}\right] \in \mathrm{R}^{n(k-i) \times n k} .
\end{aligned}
$$

With these notations one gets the following relations deduced from the Taylor series:

$$
\begin{aligned}
& \tilde{x}(t-h)=\tilde{x}(t)-h \tilde{r}_{1}(t) \\
& E_{1} \tilde{r}_{1}(t)=F_{1} \tilde{x}(t)-\frac{h}{2} \tilde{r}_{2}(t) \\
& \quad \vdots \\
& E_{k-1} \tilde{r}_{k-1}(t)=F_{k-1} \tilde{x}(t)-\frac{h}{k} \tilde{r}_{k}(t)
\end{aligned}
$$


along with the relations $(i=1 \ldots k)$

$$
\begin{gathered}
\tilde{x}(t-h)=\delta_{h}[\tilde{x}(t)] \\
\tilde{r}_{1}(t)=\delta_{h_{1}^{*}}\left[\tilde{z}_{1}(t)\right] \\
\vdots \\
\tilde{r}_{k}(t)=\delta_{h_{k}^{*}}\left[\tilde{z}_{k}(t)\right]
\end{gathered}
$$

where $\tilde{z}_{i}(t)=F_{i-1} \dot{\tilde{x}}(t)$. At this stage we have exhibited all relations involved by the Taylor series up to the power $k$. All produced operators $\delta_{h_{i}^{*}}$ have the property to be norm bounded by $1:\left|e^{-s h_{i}^{*}}\right| \leq 1$ for all $s \in \mathrm{C}^{+}$. In order to derive stability conditions it is now needed to introduce the dynamical system. Again, as the delay operator is constant one gets that

$$
\begin{aligned}
& \dot{x}(t)=A x(t)+A_{d} x(t-h) \\
& \ddot{x}(t)=A \dot{x}(t)+A_{d} \dot{x}(t-h) \\
& \quad \vdots \\
& x^{(k)}(t)=A x^{(k-1)}(t)+A_{d} x^{(k-1)}(t-h)
\end{aligned}
$$

which reads in a compact form as

$$
\dot{\tilde{x}}(t)=1_{k} \otimes A \tilde{x}(t)+1_{k} \otimes A_{d} \tilde{x}(t-h)
$$

where $E_{1} \dot{\tilde{x}}(t)=F_{1} \tilde{x}(t)$.

All these relations may be gathered in a unique representation as of Figure 1 defining the vectors

$$
z(t)=\left(\begin{array}{c}
\dot{\tilde{x}}(t) \\
\tilde{x}(t) \\
\tilde{z}_{1}(t) \\
\tilde{z}_{2}(t) \\
\vdots \\
\tilde{z}_{k}(t)
\end{array}\right), w(t)=\left(\begin{array}{c}
\tilde{x}(t) \\
\tilde{x}(t-h) \\
\tilde{r}_{1}(t) \\
\tilde{r}_{2}(t) \\
\vdots \\
\tilde{r}_{k}(t)
\end{array}\right)
$$

the system matrices

$$
\begin{aligned}
& \mathcal{E}= \\
& {\left[\begin{array}{cccccc}
1_{n k} & 0 & 0 & 0 & \cdots & 0 \\
0 & 1_{n k} & 0 & 0 & \cdots & 0 \\
E_{1} & -F_{1} & 0 & 0 & \cdots & 0 \\
\hline 1_{n k} & 0 & -1_{n k} & 0 & \cdots & 0 \\
F_{1} & 0 & 0 & -1_{n(k-1)} & \cdots & 0 \\
\vdots & & & & \ddots & \\
F_{k-1} & 0 & 0 & 0 & \cdots & -1_{n} \\
\hline 0 & 0 & 0 & 0 & \cdots & 0 \\
\vdots & & & & & \vdots \\
0 & 0 & 0 & 0 & \cdots & 0
\end{array}\right]}
\end{aligned}
$$

$$
\begin{aligned}
& \mathcal{A}= \\
& {\left[\begin{array}{cccccc}
1_{k} \otimes A & 1_{k} \otimes A_{d} & 0 & 0 & \ldots & 0 \\
1_{n k} & 0 & 0 & 0 & \ldots & 0 \\
0 & 0 & 0 & 0 & \ldots & 0 \\
\hline 0 & 0 & 0 & 0 & \cdots & 0 \\
\vdots & & & & & \vdots \\
0 & 0 & 0 & 0 & \ldots & 0 \\
\hline-1_{n k} & 1_{n k} & h 1_{n k} & 0 & \cdots & 0 \\
-F_{1} & 0 & E_{1} & \frac{h}{2} 1_{n(k-1)} & \ldots & 0 \\
\vdots & & & \ddots & \ddots & \\
-F_{k-1} & 0 & 0 & \ldots & E_{k-1} & \frac{h}{k} 1_{n}
\end{array}\right]}
\end{aligned}
$$


along with the set

$$
\mathbb{W}=\left\{\nabla=\operatorname{diag}\left(\begin{array}{l}
s^{-1} 1_{n k} \\
e^{-s h} 1_{n k} \\
e^{-s h_{1}^{*}} 1_{n k} \\
e^{-s h_{2}^{*}} 1_{n(k-1)} \\
\vdots \\
e^{-s h_{k}^{*}} 1_{n}
\end{array}\right), s \in \mathrm{C}^{+}\right\}
$$

Theorem 2 Let an integer $k \geq 1$. If there exist symmetric positive definite matrices $P \in \mathrm{R}^{k n \times k n}, Q \in$ $\mathrm{R}^{k n \times k n}, R_{1} \in \mathrm{R}^{k n \times k n}, R_{2} \in \mathrm{R}^{(k-1) n \times(k-1) n}, \ldots R_{k} \in \mathrm{R}^{n \times n}$ such that the LMI (1) holds with $\mathcal{E}$ and $\mathcal{A}$ defined in (7) and (8) respectively and

$$
\Theta=\left[\begin{array}{c}
\operatorname{diag}\left(\begin{array}{c}
0 \\
-Q \\
-R_{1} \\
-R_{2} \\
\vdots \\
-R_{k}
\end{array}\right) \\
\operatorname{diag}\left(\begin{array}{c}
-P \\
-P \\
0 \\
0 \\
0 \\
\vdots \\
0
\end{array}\right)
\end{array}\right.
$$

then system (3) is asymptotically stable for the delay $h$.

Proof: Due to Theorem 1 is suffices to prove that inequality (2) holds for all $\nabla$ of the set (9):

$$
\left[\begin{array}{cc}
1 & \nabla^{*}
\end{array}\right] \Theta\left[\begin{array}{c}
1 \\
\nabla
\end{array}\right]=\operatorname{diag}\left(\begin{array}{c}
-\left(s^{-1}+s^{-*}\right) P \\
\left(e^{-s h}-1\right) Q \\
\left(e^{-s h_{1}^{*}}-1\right) R_{1} \\
\left(e^{-s h_{2}^{*}}-1\right) R_{2} \\
\vdots \\
\left(e^{-s h_{k}^{*}}-1\right) R_{k}
\end{array}\right)
$$

All elements of this block-diagonal matrix are negative semi-definite for all $s \in \mathrm{C}^{+}$and any positive value of $h$ and $h_{i}^{*}$.

Quadratic separation used here avoids to exhibit a Lyapunov functional for proving stability. Nevertheless, the result is necessarily related to some implicit Lyapunov functional. In order to give an insight of that functional note that condition (1) is equivalent to writing that

$$
\left(\begin{array}{cc}
z^{T}(t) & w^{T}(t)
\end{array}\right) \Theta\left(\begin{array}{c}
z(t) \\
w(t)
\end{array}\right)>0
$$

for all trajectories of the system $\mathcal{E} z(t)=\mathcal{A} w(t)$. Take not identically zero signals as defined in (6), it reads

$$
\begin{aligned}
& \dot{\tilde{x}}^{T}(t) P \tilde{x}(t)+\tilde{x}^{T}(t) P \dot{\tilde{x}}(t) \\
& +\tilde{x}^{T}(t) Q \tilde{x}(t)-\tilde{x}^{T}(t-h) Q \tilde{x}(t-h) \\
& +\sum_{i=1}^{k} \tilde{z}_{i}^{T}(t) R_{i} \tilde{z}_{i}(t)-\tilde{r}_{i}^{T}(t) R_{i} \tilde{r}_{i}(t)<0 .
\end{aligned}
$$

The first and second rows of that inequality are respectively exactly the time derivative of

$$
V_{P}\left(x_{t}\right)=\tilde{x}^{T}(t) P \tilde{x}(t)
$$




$$
V_{Q}\left(x_{t}\right)=\int_{t-h}^{t} \tilde{x}^{T}(\tau) Q \tilde{x}(\tau) d \tau
$$

which are positive definite and are implicit functionals of the state. As for the third row of (10), it is a quadratic functional of $\dot{\tilde{x}}(t), \tilde{x}(t)$ and $\tilde{x}(t-h)$. At this stage we have not succeeded to express it directly as the derivative of some positive definite functional.

Proposition 1 If the conditions of Theorem 2 have a feasible solution for some integer $k$, then the conditions have a solutions for any $\hat{k} \geq k$.

Proof: Operating with the signals $z(t)$ and $w(t)$ as upper, and by small perturbation of the definite positive inequality (1) one can prove that there exist small positive scalars $\epsilon_{P}, \epsilon_{Q}, \epsilon_{1} \ldots \epsilon_{k}$ and $\epsilon_{k+1}$ such that

$$
\begin{aligned}
& \dot{\tilde{x}}^{T}(t) P \tilde{x}(t)+\tilde{x}^{T}(t) P \dot{\tilde{x}}(t)+2 \epsilon_{P} x^{(k)^{T}} x^{(k+1)} \\
& +\tilde{x}^{T}(t) Q \tilde{x}(t)-\tilde{x}^{T}(t-h) Q \tilde{x}(t-h) \\
& +\epsilon_{Q}\left(\left\|x^{(k)}(t)\right\|^{2}-\left\|x^{(k)}(t-h)\right\|^{2}\right) \\
& +\sum_{i=1}^{k} \tilde{z}_{i}^{T}(t) R_{i} \tilde{z}_{i}(t)-\tilde{r}_{i}^{T}(t) R_{i} \tilde{r}_{i}(t) \\
& +\sum_{i=1}^{k+1} \epsilon_{i}\left(\left\|x^{(k+1)}(t)\right\|^{2}-\left\|r_{i}^{(k+1-i)}(t)\right\|^{2}\right) \\
& \quad<0 .
\end{aligned}
$$

This inequality conversely proves that conditions of Theorem 2 hold for the index $k+1$ when choosing

$$
\begin{gathered}
\hat{P}=\left[\begin{array}{cc}
\epsilon_{P} 1_{n} & 0 \\
0 & P
\end{array}\right], \hat{Q}=\left[\begin{array}{cc}
\epsilon_{Q} 1_{n} & 0 \\
0 & Q
\end{array}\right] \\
\hat{R}_{1}=\left[\begin{array}{cc}
\epsilon_{1} 1_{n} & 0 \\
0 & R_{1}
\end{array}\right] \quad \ldots \quad \hat{R}_{k}=\left[\begin{array}{cc}
\epsilon_{k} 1_{n} & 0 \\
0 & R_{k}
\end{array}\right]
\end{gathered}
$$

and $\hat{R}_{k+1}=\epsilon_{k+1} 1_{n}$.

Proposition 1 indicates that the sequence of LMI problems generated by Theorem 2 are less and less conservative as $k$ grows. This convergence will be illustrated on examples in section V. It will be seen that although the first steps give good results in terms of conservatism, the convergence to exactness is not achieved even for $k=10$ for which the numerical complexity becomes prohibitive. Therefore, form a numerical point view, the exposed results cannot be considered as approximating asymptotically the exact problem, although, it may be true theoretically.

\section{Robust stability with respect to uncertain delay}

One significant characteristic of the quadratic separation framework is that is allows to handle simultaneously several uncertain operators. One application of that is proving robustness and we focus now on systems with uncertain non-small delay. $h$ is considered as constant and unknown in some interval $[\underline{h} \bar{h}]$. Starting from the results of the previous section, for the case $k=2$ for simplicity, the problem is demonstrated to have LMI solutions.

For $k=2$, the delay $h$ enters the expanded model of the previous section as two affine products $h \tilde{r}_{1}(t)$ and $\frac{1}{2} h \tilde{r}_{2}(t)$. Define new exogenous signals such that

$$
\tilde{w}_{1}=h \tilde{r}_{1}(t), \quad \tilde{w}_{2}=h \tilde{r}_{2}(t)
$$

then a new expanded model for the system writes as Figure 1 with the newly defined the vectors

$$
z(t)=\left(\begin{array}{c}
\dot{\tilde{x}}(t) \\
\tilde{x}(t) \\
\tilde{z}_{1}(t) \\
\tilde{z}_{2}(t) \\
\tilde{r}_{1}(t) \\
\tilde{r}_{2}(t)
\end{array}\right), w(t)=\left(\begin{array}{c}
\tilde{x}(t) \\
\tilde{x}(t-h) \\
\tilde{r}_{1}(t) \\
\tilde{r}_{2}(t) \\
\tilde{w}_{1}(t) \\
\tilde{w}_{2}(t)
\end{array}\right)
$$


the newly defined system matrices

$$
\mathcal{E}=\left[\begin{array}{cccccc}
1_{2 n} & 0 & 0 & 0 & 0 & 0 \\
0 & 1_{2 n} & 0 & 0 & 0 & 0 \\
E_{1} & -F_{1} & 0 & 0 & 0 & 0 \\
\hline 1_{2 n} & 0 & -1_{2 n} & 0 & 0 & 0 \\
F_{1} & 0 & 0 & -1_{n} & 0 & 0 \\
\hline 0 & 0 & 0 & 0 & 0 & 0 \\
0 & 0 & 0 & 0 & 0 & 0 \\
\hline 0 & 0 & 0 & 0 & 1_{2 n} & 0 \\
0 & 0 & 0 & 0 & 0 & 1_{n}
\end{array}\right]
$$

$$
\mathcal{A}=\left[\begin{array}{cccccc}
1_{2} \otimes A & 1_{2} \otimes A_{d} & 0 & 0 & 0 & 0 \\
1_{2 n} & 0 & 0 & 0 & 0 & 0 \\
0 & 0 & 0 & 0 & 0 & 0 \\
\hline 0 & 0 & 0 & 0 & 0 & 0 \\
0 & 0 & 0 & 0 & 0 & 0 \\
\hline-1_{2 n} & 1_{2 n} & 0 & 0 & 1_{2 n} & 0 \\
-F_{1} & 0 & E_{1} & 0 & 0 & \frac{1}{2} 1_{n} \\
\hline 0 & 0 & 1_{2 n} & 0 & 0 & 0 \\
0 & 0 & 0 & 1_{n} & 0 & 0
\end{array}\right]
$$

along with the set

$$
\mathbb{W}=\left\{\nabla=\operatorname{diag}\left(\begin{array}{l}
s^{-1} 1_{2 n} \\
e^{-s h} 1_{2 n} \\
e^{-s h_{1}^{*} 1_{2 n}} \\
e^{-s h_{2}^{*} 1_{n}} \\
h 1_{3 n}
\end{array}\right), \quad s \in\left[\begin{array}{l}
\mathrm{C}^{+} \\
\underline{h}]
\end{array}\right\} .\right.
$$

Theorem 3 If there exist symmetric positive definite matrices $P \in \mathrm{R}^{2 n \times 2 n}, Q \in \mathrm{R}^{2 n \times 2 n}, R_{1} \in \mathrm{R}^{2 n \times 2 n}$, $R_{2} \in \mathrm{R}^{n \times n}, S_{3} \in \mathrm{R}^{3 n \times 3 n}$, a symmetric matrix $S_{1} \in \mathrm{R}^{3 n \times 3 n}$ and a square matrix $S_{2} \in \mathrm{R}^{3 n \times 3 n}$, such that the LMI (1) holds with $\mathcal{E}$ and $\mathcal{A}$ defined in (12) and (13) respectively and

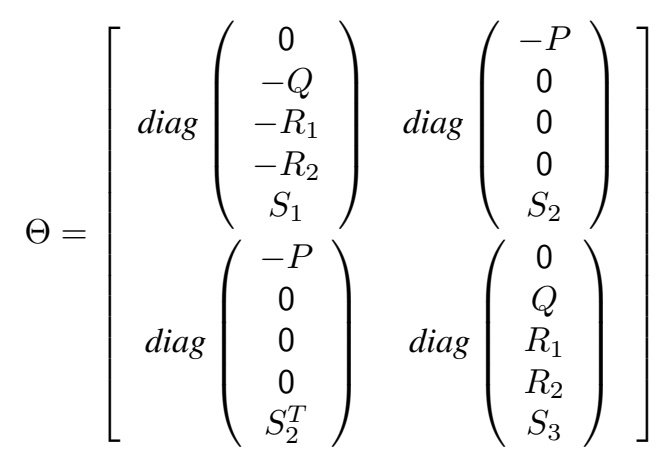

with the additional constraints

$$
\begin{aligned}
& S_{1}+\underline{h}\left(S_{2}+S_{2}^{T}\right)+\underline{h}^{2} S_{3} \leq 0 \\
& S_{1}+\bar{h}\left(S_{2}+S_{2}^{T}\right)+\bar{h}^{2} S_{3} \leq 0
\end{aligned}
$$

then system (3) is asymptotically stable for any delay $h \in[\underline{h} \bar{h}]$.

Proof: Due to Theorem 1 is suffices to prove that the inequality (2) holds for all $\nabla$ of the set (14):

$$
\left[\begin{array}{c}
1 \\
\nabla
\end{array}\right]^{*} \Theta\left[\begin{array}{c}
1 \\
\nabla
\end{array}\right]=\operatorname{diag}\left(\begin{array}{c}
-\left(s^{-1}+s^{-*}\right) P \\
\left(e^{-s h}-1\right) Q \\
\left(e^{-s h_{1}^{*}}-1\right) R_{1} \\
\left(e^{-s h_{2}^{*}}-1\right) R_{2} \\
S_{1}+h\left(S_{2}+S_{2}^{T}\right)+h^{2} S_{3}
\end{array}\right)
$$


The four first terms of this block-diagonal matrix are negative semi-definite for all $s \in \mathrm{C}^{+}$whatever the positive values of $h, h_{i}^{*}$. As $S_{3}$ is chosen positive definite, the last term is convex with respect to $h$. Being negative semi-definite on the extrema of the interval $[\underline{h} \bar{h}]$ it is negative semi-definite for all the interval.

\section{Numerical examples}

The results are evaluated on a simple academic example taken from [10] for which the analytical bounds on stabilizing delays is known. The system is such that

$$
\ddot{y}(t)-0.1 \dot{y}(t)+2 y(t)=u(t)
$$

Following $[10,15,13]$, we aim to stabilize the system using a static delayed output-feedback $u(t)=$ $k y(t-h)$. Choosing $k=1$, we get the following state space model:

$$
\dot{x}(t)=\left[\begin{array}{cc}
0 & 1 \\
-2 & 0.1
\end{array}\right] x(t)+\left[\begin{array}{ll}
0 & 0 \\
1 & 0
\end{array}\right] x(t-h) .
$$

Clearly, for large values of $h$ and for $h=0$ the system is unstable (Eigenvalues of $A$ and $A+A_{d}$ are respectively $\{0.0500+1.4133 i, 0.0500-1.4133 i\}$ and $\{0.0500+0.9987 i, 0.0500-0.9987 i\}$. Classical delay-dependent techniques like [7, 20,6] cannot be applied. Note also that techniques developed for non-small delays of $[4,18]$ fail as well. As for results of Theorem 2 their efficiency is shown in Table 1.

For Taylor series of the delay operator taken up to the power $k$ with $k$ ranging from 0 up to 10 , we have estimated by dichotomy the interval of stabilizing delays. $\varnothing$ indicates that the method fails which is expected for $k=0$ (that result is applicable only to systems stable whatever positive delay) and for $k=1$ (result applicable only to systems stable for zero delay). The table gives as well the number of variables involved in the LMI problems. The numerical complexity grows significantly as $k$ goes to infinity nevertheless one can see that conservatism is already quite low for small values of $k$. The open interval ] 0.100168261 .7178 [ of asymptotically stabilizing delays is quite well approached.

Table 1: Interval of stabilizing delays for system (16) obtained by dichotomy with the use of Theorem 2

\begin{tabular}{lllr}
\hline & $h_{\min }$ & $h_{\max }$ & Number of decision variables \\
\hline$k=0$ & $\varnothing$ & $\emptyset$ & 6 \\
$k=1$ & $\varnothing$ & $\emptyset$ & 9 \\
$k=2$ & 0.1009 & 1.423 & 33 \\
$k=3$ & 0.1001684 & 1.440 & 76 \\
$k=4$ & 0.1001684 & 1.440 & 142 \\
$k=5$ & 0.1001684 & 1.440 & 235 \\
$k=6$ & 0.10016832 & 1.634 & 359 \\
$k=7$ & 0.10016832 & 1.704 & 518 \\
$k=8$ & 0.10016832 & 1.704 & 716 \\
$k=9$ & 0.10016832 & 1.704 & 957 \\
$k=10$ & 0.10016832 & 1.710 & 1245 \\
$k=11$ & 0.10016832 & 1.711 & 1584 \\
analytical & 0.10016826 & 1.7178 & - \\
\hline
\end{tabular}

But these results do not prove formally the stability for all values of the intervals. One can argue that stability is only proved for the selected values and may not be true for all values inside the interval. Apply for that purpose Theorem 3. As this theorem is an extension of Theorem 2 for the case $k=2$, one cannot expect to prove stability for any interval outside [0.1009 1.423]. First we attempt to apply Theorem 3 to that interval and the LMIs are found infeasible indicating the conservatism of the result. Then, the LMIs 
of Theorem 3 are tested for smaller intervals

$$
[0.10090 .103],[0.1030 .33],[0.31 .34],[1.341 .423] \text {. }
$$

and prove to be feasible. The system is stable whatever the delay in any of these intervals, proving therefore stability for the whole interval [0.1009 1.423]. Compared to the LMIs involved in Theorem 2, the LMI problems solved using Theorem 3 have 111 decision variables. At the expense of increasing from 32 to 111 the number of variables, robust stability is proved for any uncertain delay lying inside [0.1009 1.423].

To confirm the numerical results a second example is tested. The system matrices are as follows

$$
A=\left[\begin{array}{cc}
0 & 1 \\
-2 & 0
\end{array}\right], A_{d}=\left[\begin{array}{cc}
0 & 0 \\
0.4 & 0
\end{array}\right]
$$

The system is not asymptotically stable for $h=0$ and results of $[7,20,6,4,18]$ therefore fail. Given in [15] using analytical methods, it is known that for $h$ taken in the open interval ]0 2.027[ the system is asymptotically stable. As for the previous example several sub intervals of that were tested using Theorem 3. The LMIs are found feasible for all the intervals

$$
\left[10^{-5} 0.03\right],[0.030 .67],[0.671 .52],[1.521 .53]
$$

therefore proving asymptotic stability for any $h \in\left[10^{-5} 1.53\right]$. The remaining gap with the interval obtained using analytical methods illustrates the conservatism remaining in the method. That conservatism may be reduced by applying the robust methodology on results of Theorem 2 for larger values of $k$.

\section{Conclusion}

The paper exposes new ways for stability analysis of a linear time-delay system with non-small delay. The approach relies on the use of quadratic separation framework combined with a Taylor series approximation of the delay operator. A sequence of LMI problems can be generated and they are proved to have decreasing conservatism. Extensions to robust stability analysis with respect to uncertain non-small delay are given. All these results show their efficiency on numerical examples. A numerical comparison with results of $[9,14]$ will be carried out for the final version of the paper.

Future work will consider other robustness issues such as robustness with respect to both time-delay uncertainties and parametric uncertainties and such as robustness with respect to uncertain time-vaying delays.

\section{References}

[1] G. Abdallah, P. Dorato, J. Benitez-Read, and R. Byrne. Delayed positive feedback can stabilize oscillatory systems. In ACC'93, American Control Conference, pages 3106-3107, 1993.

[2] P.A. Bliman. Lyapunov equation for the stability of linear delay systems of retarded and neutral type. IEEE Trans. Aut. Control, 47(2):327-335, Feb 2002.

[3] J. Chiasson and C. T. Abdallah. Robust stability of time delay systems: Theory. In 3rd IFAC Workshop on Time Delay Systems, Santa Fe, December 2001.

[4] E. Fridman. A new Lyapunov technique for robust control of systems with uncertain non-small delays. IMA Journal of Math. Control and Information, 23, 2006.

[5] E. Fridman and U. Shaked. A descriptor system approach to $H_{\infty}$ control of linear time-delay systems. IEEE Trans. Aut. Control, 47(2):253-270, 2002.

[6] E. Fridman and U. Shaked. Delay-dependent stability and $h_{\infty}$ control: constant and time varying delay. International journal of control, 76(1):48-60, 2003. 
[7] F. Gouaisbaut and D. Peaucelle. Delay-dependent robust stability of time delay systems. In ROCOND, Toulouse, July 2006.

[8] F. Gouaisbaut and D. Peaucelle. A note on the stability of time delay systems. In ROCOND, Toulouse, July 2006.

[9] K. Gu. Discretized LMI set in the stability problem of linear uncertain time-delay systems. Internat. J. Control, 68:923-934, 1997.

[10] K. Gu, K.L. Kharitonov, and J. Chen. Stability of Time-Delay Systems. Control Engineering Series. Birkhauser, Boston USA, 2003.

[11] T. Iwasaki and S. Hara. Well-posedness of feedback systems: Insights into exact robustness analysis and approximate computations. IEEE Trans. Aut. Control, 43(5):619-630, 1998.

[12] V. Kharitonov and A. Zhabko. Lyapunov-Krasovskii approach to the robust stability analysis of timedelay systems. Automatica, 39:15-20, 2003.

[13] V.L. Kharitonov and S.-I. Niculescu. On the stability of linear systems with uncertain delay. IEEE Trans. Aut. Control, 48(1):127-132, 2003.

[14] C.R. Knospe and M. Roozbehani. Stability of linear systems with interval time-delay. In American Control Conference, pages 1458-1463, Denver, Colorado, June 2003.

[15] S.I. Niculescu, K. Gu, and C.T. Abdallah. Some remarks on the delay stabilizing effect in SISO systems. In American Control Conference, Denver, June 2003.

[16] D. Peaucelle, D. Henrion, and D. Arzelier. Quadratic separation for feedback connection of an uncertain matrix and an implicit linear transformation. In 16th IFAC World Congress, Prague, Czech Republic, July 2005.

[17] M.G. Safonov. Stability and Robustness of Multivariable Feedback Systems. Signal Processing, Optimization, and Control. MIT Press, 1980.

[18] V. Suplin, E. Fridman, and U. Shaked. Input-output approach to the control of time-delayed systems. In IEEE International Symposium on, Mediterrean Conference on Control and Automation, June 2005 .

[19] E. T. Whittaker and G.N. Watson. A Course in Modern Analysis. Cambridge University Press,England, 4th ed. edition, 1990.

[20] M. Wu, Y. He, J.H. She, and G.P Liu. Delay dependent criteria for robust stability of time-varying delay systems. Automatica, 40:1435-1439, 2004.

[21] J. Zhang, C.R. Knospe, and P. Tsiotras. Stability of time-delay systems: Equivalence between Lyapunov and scaled small-gain conditions. IEEE Trans. Aut. Control, 46(3):482-486, March 2001. 